\title{
Solving Integer Programming Problems by Hybrid Bat Algorithm and Direct Search Method
}

\author{
Ahmed F Ali', and Mohamed A Tawhid ${ }^{2,3^{*}}$ \\ ${ }^{1}$ Faculty of Computers \& Informatics, Department of Computer Science, Suez Canal University, Egypt \\ ${ }^{2}$ Faculty of Science, Department of Mathematics and Statistics, Thompson Rivers University, Canada \\ ${ }^{3}$ Faculty of Science, Department of Mathematics and Computer Science, Alexandria University, Egypt
}

\begin{abstract}
The goal of this work is to suggest a new hybrid algorithm to solve integer programming by incorporating the bat algorithm with direct search methods. The suggested algorithm is named hybrid bat direct search algorithm (HBDS). In HBDS, the global diversification and the local intensification process are balanced. The bat algorithm has a good capability to make intensification and diversification search. The intensification ability of the suggested algorithm is increased by employing the pattern search method as a local search method instead of the random walk method in the classic bat algorithm. In the final stage of the algorithm, the Nelder-Mead method is used to improve the best found solution from the bat and pattern search method instead of running the algorithm more iterations without any enhancements in the fitness function value. The performance of the HBDS algorithm is examined on 7 integer programming problems and compared to 10 benchmark algorithms for solving integer programming problems. The computational results show that HBDS is a promising algorithm and outperforms the other algorithms in most cases.
\end{abstract}

Keywords

Bat algorithm, Direct search methods, Pattern search, Nelder-Mead method, Integer programming problems

\section{Introduction}

Integer programming (IP) appear closely in every research area in applied operations research and mathematical programming. Variety of many real life applications for IP problems such as, scheduling problem, VLSI (very large scale integration) circuits design problems, engineering design problems, warehouse location problem, robot path planning problems, [1-3] can be formulated as IP problems.

On one hand, traditional integer programming methods such as dynamic programming or branch and bound have high computational cost, because they examine a search tree that has hundreds or more nodes when large scale real-life problems are considered. On the other hand, heuristic and metaheuritsic methods can be applied for solving integer programming problems. Swarm intelligence (SI) algorithms are novel meta-heuristics algorithms, which find their inspiration from the behavior of a group of social organisms. These algorithms are used to solve global optimization problems and their applications such as ant colony optimization (ACO) $[4,5]$ artificial bee colony [6] particle swarm optimization (PSO) [7-9], bacterial foraging [10], bat algorithm [11,12], bee colony optimization (BCO) [13], wolf search [14], cat swarm [15], Cuckoo search [16], firefly algorithm [17], fish swarm/school [18], etc.

These algorithms have been commonly adopted to

*Corresponding author: Mohamed A Tawhid, Faculty of Science, Department of Mathematics and Statistics, Thompson Rivers University, Kam-loops, BC V2C 0C8, Canada; Faculty of Science, Department of Mathematics and Computer Science, Alexandria University, Mo-haram Bey 21511, Alexandria, Egypt, Tel: 250-377-6041, Fax: 250-371-5675, E-mail: Mtawhid@tru.ca

Accepted: July 23, 2018; Published online: July 25, 2018

Citation: Ali AF, Tawhid MA (2018) Solving Integer Programming Problems by Hybrid Bat Algorithm and Direct Search Method. Trends Artif Intell 2(1):46-59.

Copyright: @ 2018 Ali AF, et al. This is an open-access article distributed under the terms of the Creative Commons Attribution License, which permits unrestricted use, distribution, and reproduction in any medium, provided the original author and source are credited. 
Citation: Ali AF, Tawhid MA (2018) Solving Integer Programming Problems by Hybrid Bat Algorithm and Direct Search Method. Trends Artif Intell 2(1):46-59.

solve unconstrained and constrained optimization problems and their applications, nevertheless there are not many SI algorithms used to solve integer programming problems [19-22]. There are some efforts to use some of meta-heuristics algorithms to solve integer programming problems such as social spider optimization [23], gravitational search algorithm [20], particle swarm optimization algorithm [7,24], firefly algorithm [25,26], cuckoo search algorithm [27-29], artificial bee colony algorithm [30-32], ant colony algorithm [33], and simulated annealing [19].

Bat algorithm (BA) is a recent population based algorithm inspired from the echolocation behavior of the microbats [12]. BA is capable to balance the global diversification and the local intensification during the search process. Because of the powerful performance of the BA, it has been used by many researchers to solve diverse applications, for example, Lin, et al. [34] used parameter estimation in dynamic biological systems using a chaotic bat algorithm by integrating Levy flights and chaotic maps. Zhang and Wang [35] improved the diversity of solutions by using the mutation with bat algorithm for image matching. Yang [36] applied BA to solve multi-objective optimization and benchmark engineering problems. Komarasamy and Wahi [37] integrated $\mathrm{K}-\mathrm{means}$ and bat algorithm (KMBA) for efficient clustering. Nakamura, et al. [38] developed a discrete version of bat algorithm to solve classifications and feature selection problems. Xie, et al. [39] presented a variant of bat algorithm integrating Levy flights and differential operator to solve function optimization problems. In addition, Wang and Guo [40] integrated harmony search with bat algorithm and generated a hybrid bat algorithm for numerical optimization of function benchmarks.

The purpose of this paper is to avoid the slow convergence of the BA and avoid trapping in local minima. In order to solve these two issues, we suggest a new hybrid bat algorithm with direct search methods to solve integer programming problems [41]. The suggested algorithm is named hybrid bat direct search algorithm (HBDS). In HBDS, the pattern search is employed as a local search method to exploit the search around the best found solution at each iteration and in the final stage of the algorithm, the Nelder-Mead method is called to enhance the best obtained solution from the bat and pattern search method. Using the Nelder-Mead method can hasten the search and evade running the algorithm with more iterations without any enhancements in the results.

The rest of this paper is structured as follows. In Section 4 , the integer programming problems and the applied direct search methods, the classic BA are described. In Section 5, the main concepts of the suggested HBDS algorithm are given. The numerical experimental and re- sults are shown in Section 6. Finally, the conclusion and future work are presented in Section 7.

\section{Definition of the Problems and an Overview of the Applied Algorithms}

In this section and its subsections, the definitions of the integer programming problems are presented and an overview of the $\mathrm{BA}$ and the pattern search method is given as follows.

\section{The integer programming problem definition}

An integer programming problem is a mathematical optimization problem in which all of the variables are restricted to be integers. The unconstrained integer programming problem can be defined as follows.

$$
\min f(x), x \in S \subseteq \mathbb{Z}^{n},
$$

Where $\mathbb{Z}$ is the set of integer variables, $S$ is a not necessarily bounded set.

\section{Pattern search method}

The authors in [42] introduced the pattern search method (PS). Pattern search method is an applied direct search method to solve a global optimization problems. In direct search method, there is no need for any information about the gradient of the objective function to solve optimization problem. PS method has two type of moves, the exploratory moves and the pattern moves. In the exploratory moves a coordinate search is used around a chosen solution with a step length of $\Delta$ in Algorithm 1 . The exploratory move is considered successful if the function value of the new solution is better than the current solution. Otherwise, the step length is reduced. If the exploratory move is successful, then the pattern search is used in order to produce the iterate solution. If the iterate solution is better than the current solution, then exploratory move is used on the iterate solution and the iterate solution is accepted as a new solution. Otherwise, if the exploratory move is unsuccessful, the pattern move is rejected and the step length $\Delta$ is decreased. The operation is repeated until stopping criteria are satisfied. The algorithm of Hook and Jeeves $(\mathrm{HJ})$ pattern search and the main steps of it are outlined in Algorithm 2. The parameters in Algorithms 1, 2 are outlined in Table 1.

\section{A Nelder-Mead method}

The main steps of the Nelder-Mead (NM) algorithm

Table 1: The parameters of the pattern search algorithm.

\begin{tabular}{|l|l|}
\hline Parameter & Definition \\
\hline$\Delta_{0}$ & Initial mesh size \\
\hline$d$ & Variable mesh size \\
\hline$\sigma$ & Reduction factor of mesh size \\
\hline$m$ & Pattern search repetition number \\
\hline$\varepsilon$ & Tolerance \\
\hline
\end{tabular}


Citation: Ali AF, Tawhid MA (2018) Solving Integer Programming Problems by Hybrid Bat Algorithm and Direct Search Method. Trends Artif Intell 2(1):46-59.

[43] in two dimensions is presented as follows. There are four scalar parameters that represent the main parameters of the NM algorithm such as: Coefficients of reflection $\rho$, expansion $\chi$, contraction $\tau$, and shrinkage Table 1 .

\section{Algorithm 1: Exploratory search}

INPUT: Get the values of $x^{0}, k, \Delta_{0}, d$

OUTPUT: New base point $x^{k}$

1. Set $i=1$

2. Set $k=1$

3. repeat

4. Set $x_{i}^{k}=x_{i}^{k-1}+\Delta_{k-1} x_{i}^{k-1}$

5. if $f\left(x_{i}^{k}\right)<f\left(x_{i}^{k-1}\right)$ then

6. Set $x_{i}^{k+1}=x_{i}^{k}$

7. end if

8. Set $i=i+1$

9. Set $k=k+1$

10.until $i>d$

$\phi$ where $\rho>0, \chi>1,0<\tau<1$, and $0<\phi<1$. The main steps of the NM algorithm are shown in Algorithm 3.

\section{Overview of the Bat algorithm}

In this section, an overview of the main concepts and structure of the $\mathrm{BA}$ is given.

Main concepts: Bat algorithm (BA) is a population based metaheuristic algorithm, was developed by XinShe Yang in 2010 [12]. BA is based on the echolocation of microbats, which use a type of sonar (echolocation) to detect prey and avoid obstacles in the dark. The main advantage of the BA is that it can provide a fast convergence at a very initial stage by switching from diversification to intensification, however, switching from diversification to intensification quickly may lead to stagnation after some initial stage.

The rules of the BA: Based on the bat characteristics, Xin-She Yang developed the bat algorithm with the following rules.

- All bats can distinguish between pray and barriers/obstacles by using echolocation to sense distance.

Algorithm 2: The basic pattern search algorithm.

INPUT: Get the values of $x$.

OUTPUT: Best solution $x^{*}$.

1. Set the values of the initial values of the mesh size $\Delta_{0}$, reduction factor of mesh size $\sigma$ and termination pa- rameter $\varepsilon$

\section{Set $k=1$ Parameter setting\}}

3. Set the starting base point $x^{k-1}\{$ Initial solution $\}$

4. repeat

5. Perform exploratory search as shown in Algorithm 1

6. if exploratory move success then

7. Go to 16

8. else

9. if $\|\Delta\|<$, then

10. Stop the search and the current point is $x^{*}$

11.else

12. Set $\Delta_{k}=\sigma \Delta_{k-1}\{$ Incremental change reduction $\}$

13. Go to 5

\section{4.end if}

15.end if

16. Perform pattern move, where $x_{p}^{k+1}=x^{k}+\left(x^{k}-x^{k-1}\right)$

17. Perform exploratory move with $x_{p}$ as the base point

18. Set $x^{k+1}$ equal to the output result exploratory move

19.if $f\left(x_{p}^{k+1}\right)<f\left(x^{k}\right)$ then

20. Set $x^{k-1}=x^{k}$

21. Set $x^{k}=x^{k+1}$ New base point $\}$

22. Go to 16

23.else

24. Go to 9 \{The pattern move fails\}

25.end if

26. Set $k=k+1$

27.until $k>m$

Algorithm 3: The Nelder-Mead Algorithm.

1. Let $x_{i}$ denote the list of vertices in the current simplex, $i=1, \ldots, n+1$.

2. Order. Order and re-label the $n+1$ vertices from lowest function value $f\left(x_{1}\right)$ to highest function value $f\left(x_{n+1}\right)$ so that $f\left(x_{1}\right) \leq f\left(x_{2}\right) \leq \ldots \leq f\left(x_{n+1}\right)$.

3. Reflection. Compute the reflected point $x_{r}$ by $x_{r}=\bar{x}+\rho\left(\bar{x}-x_{(n+1)}\right)$, where $\bar{x}$ is the centroid of the $\mathrm{n}$ best points,

$$
\begin{aligned}
& \bar{x}=\Sigma\left(x_{i} / n\right), i=1, \ldots, n . \\
& \text { if } f\left(x_{1}\right) \leq f\left(x_{r}\right)<f\left(x_{n}\right) \text { then }
\end{aligned}
$$


Citation: Ali AF, Tawhid MA (2018) Solving Integer Programming Problems by Hybrid Bat Algorithm and Direct Search Method. Trends Artif Intell 2(1):46-59.

Replace $x_{\mathrm{n}+1}$ with the reflected point $x_{r}$ and go to Step 7 . end if

\section{Expansion.}

if $f\left(x_{r}\right)<f\left(x_{1}\right)$ then

Computetheexpandedpoint $x_{e}$ by $x_{e}=\bar{x}+\chi\left(x_{r}-\bar{x}\right)$

end if

if $f\left(x_{e}\right)<f\left(x_{r}\right)$ then

Replace $x_{n+1}$ with $x_{e}$ and go to Step 7 .

else

Replace $x_{n+1}$ with $x_{r}$ and go to Step 7 .

\section{end if}

\section{Contraction.}

if $f\left(x_{n}\right) \leq f\left(x_{r}\right)<f\left(x_{n+1}\right)$ then

Calculate $x_{o c}=\bar{x}+\tau\left(x_{r}-\bar{x}\right)$ \{Outside contract\}

end if

if $f\left(x_{o c}\right) \leq f\left(x_{r}\right)$ then

Replace $x_{n+1}$ with $x_{o c}$ and go to Step 7 .

else

Go to Step 6.

\section{end if}

if $f\left(x_{r}\right) \geq f\left(x_{n+1}\right)$ then

Calculate $x_{i c}=\bar{x}+\tau\left(x_{n+1}-\bar{x}\right)$. \{Inside contract $\}$

\section{end if}

if $f\left(x_{i c}\right) \leq f\left(x_{n+1}\right)$ then

Replace $\mathrm{x}_{\mathrm{n}+1}$ with $\mathrm{x}_{\mathrm{ic}}$ and go to Step 7 .

else

Go to Step 6.

\section{end if}

6. Shrink. Evaluate the $\mathrm{n}$ new vertices

$$
x^{\prime}=x_{1}+\phi\left(x_{i}-x_{1}\right), i=2, \ldots, n+1 \text {. }
$$

Replace the vertices $x_{2}, \ldots, x_{n+1}$ with the new vertices $x_{2}^{\prime}, \ldots, x_{n+1}^{\prime}$.

7. Stopping Condition. Order and re-label the vertices of the new simplex as $x_{1}, x_{2}, \ldots, x_{n+1}$ such that $f\left(x_{1}\right) \leq f\left(x_{2}\right) \leq \ldots \leq f\left(x_{n+1}\right)$

if $f\left(x_{n+1}\right)-f\left(x_{1}\right)<\varepsilon$ then

Stop, where $\varepsilon>0$ is a small predetermined tolerance.

else

Go to Step 3. end if

- $\quad$ Each bat randomly moves with velocity $v_{i}$ at a position $x_{i}$ with a frequency $f_{\min }$ varying loudens $A_{0}$ and pulse emission rate $r$.

- Assume that the loudness varies from a large value $A_{0}$ to a minimum value $A_{\text {min }}$.

Bat movement: The BA is a population based method, where the population size consists of bats (solutions). Each bat (solution) in the population is randomly moving with velocity $v_{\mathrm{i}}$ and a location $x_{i}$. Also each bat is randomly assigned a frequency drawn uniformly from $\left[f_{\text {min }}\right.$, $f_{\max }$. The position of each bat in the population is updated as shown in the following equations.

$$
\begin{aligned}
& f_{i}=f_{\text {min }}+\left(f_{\text {max }}-f_{\text {min }}\right) \beta \\
& v_{i}^{t}=\mathrm{v}_{i}^{t-1}+\left(x_{i}^{t-1}-x^{*}\right) f_{i}, \\
& x_{i}^{t}=x_{i}^{t-1}+v_{i}^{t},
\end{aligned}
$$

Where $\beta \in[0,1]$ is a random vector drawn from a uniform distribution.

Variation of loudness and pulse emission rates: The loudness $A_{i}$ and the pulse rate emission $r_{i}$ are very important to let the algorithm switch between diversification and intensification process. When the bat has found its pray, the loudness decreases and the rate of pulse emission increases. The BA starts with an initial value of the loudness $A_{0}$ and the rate of pulse emission $r_{0}$, then these values are updated as shown in the following equations.

$$
\begin{aligned}
& A_{i}^{(t+1)}=\alpha A_{i}^{(t)} \\
& r_{i}^{(t)}=r_{i}^{(0)}[1-\exp (-\gamma t)]
\end{aligned}
$$

Where $\alpha \in[0,1]$ and $\gamma>0$ are constant, the parameter plays a similar role as the cooling factor in the simulated annealing algorithm.

Bat algorithm: The main steps of the classic bat algorithm are shown in Algorithm 4 and can be summarized in the following steps.

Step 1: The algorithm starts by setting the initial values of its parameters and setting zero to the main iteration counter. (Lines 1-2)

Step 2: The initial population is randomly generated by generating the initial position $x^{0}$ and the initial velocity $v^{0}$ for each bat (solution) in the population. The initial frequency $f_{i}$ is assigned to each solution in the population, where $\mathrm{f}$ is randomly generated from $\left[f_{\min }, f_{\max }\right]$. The initial population is evaluated by calculating the objective function for each solution via the initial population $f\left(x^{0}\right)$, the values of pulse rate $r_{i}$ and initial loudness $A_{i}$ where $r$ 
Citation: Ali AF, Tawhid MA (2018) Solving Integer Programming Problems by Hybrid Bat Algorithm and Direct Search Method. Trends Artif Intell 2(1):46-59.

$\in[0,1]$ and $A_{i}$ varies from a large $A_{0}$ to $A_{\text {min }}$ (Lines 3-9)

Step 3: The new population is generated by adjusting the position $x_{i}$ and the velocity $v_{i}$ for each solution in the population as in (2), (3), and (4). (Lines 12-13)

Step 4: The new population is evaluated by calculating the objective function for each solution and the best solution $x^{*}$ is selected from the population. (Lines 14-15)

Step 5: The local search method is applied in order to refine the best found solution at each iteration. (Lines 16-19)

Step 6: The new solution is accepted with some proximity depending on parameter $A_{i}$, increasing the rate of pulse emission and decreasing the loudness. The values of $A_{i}$ and $r_{i}$ are updated as in (5) and (6).

Step 7: The new population is evaluated, and the best solution is selected from the population. The operations are repeated until termination criteria are satisfied and the overall best solution is produced. (Lines 25-28)

\section{The Suggested HBDS Algorithm}

The main steps of the suggested HBDS algorithm are given in Algorithm 5 and the description of the suggested algorithm can be summarized as follows.

Algorithm 4: The bat algorithm

1. Set the initial values of the minimum frequency $f_{\text {min }}$, maximum frequency $f_{\text {max }}$, population size $P$, the loudness constant $\alpha$, the rate of pulse emission constant $\gamma$, the initial loudens $A_{0}$, the minimum loudness $A_{\text {min }}$, the initial rate of pulse emission $r^{0}$ and the maximum number of iterations $\operatorname{Max}_{i t r}$ \{Parameters initialization $\}$

\section{Set $t=0$. \{Counter initialization $\}$}

3. for $(i=1 ; i<P ; i++)$ do

4. Generate the initial bat population $x_{i}^{t}$ randomly.

5. Generate the initial bat velocities $v_{i}^{t}$ randomly.

6. Assign the initial frequency $f_{i}$ to each $x_{i}^{t}$.

7. Evaluate the initial population by calculating the objective function $f\left(x_{i}^{t}\right)$ for each solution in the population.

8. Set the initial values of the pulse rates $r_{i}$ and loudness $A_{i}$. $\{$ Population initialization $\}$

\section{9. end for}

10. repeat

11. $t=t+1$

12. Generate new bat solutions $x_{i}^{t}$ by adjusting frequency as in (4).

13. Update the bat velocities $v_{i}^{t}$ as in (2) and (3).

14. Evaluate the new population by calculating the objective function $f\left(x_{i}^{t}\right)$ for each solution in the pop- ulation.

15. Select the best solution $x^{*}$ from the population.

16. if rand $>r_{i}$ then

17. Select a solution among the best solutions.

18. Generate a local search solution around the selected best solution.

\section{9. end if}

20. Generate a random new solution.

21. if rand $<A_{i} \& f\left(x_{i}^{t}<f\left(x^{*}\right)\right)$ then

22. Accept the new solutions.

23. Increase the rate of pulse emission $r_{i}$ and reduce the loudness $A_{0}$.

24. end if

25. Evaluate the new population by calculating the objective function $f\left(x_{i}^{t}\right)$ for each solution in the population.

26. Rank the population and select the best solution $\mathrm{x}^{*}$ from the population.

27. until $\left(t>\operatorname{Max}_{i t r}\right)\{$ Termination criteria are satisfied $\}$

28. Produce the best solution.

Algorithm 5: The HBDS algorithm

1. Set the initial values of the minimum frequency $f_{\text {min }}$, the minimum loudens $A_{\text {min }}$, the rate of pulse emission constant $\gamma$, maximum frequency $f_{\max }$, the initial loudens $A_{0}$, population size $P$, the loudness constant $\alpha$, the maximum number of iterations $\operatorname{Max}_{i t r}$. and the initial rate of pulse emission $r^{0}$. \{Parameters initialization\}

2. Set $t=0$. \{Counter initialization $\}$

3. for $(i=1 ; i<P ; i++)$ do

4. Generate the initial bat population $x_{i}^{t}$ randomly.

5. Generate the initial bat velocities $v_{i}^{t}$ randomly.

6. Designate the initial frequency $f_{i}$ to each $x_{i}^{t}$.

7. Evaluate the initial population by calculating the objective function $f\left(x_{i}^{t}\right)$ for each solution in the population.

8. Set the initial values of the pulse rates $r_{i}$ and loudness $A_{i}$. $\{$ Population initialization $\}$

\section{9. end for}

10. repeat

11. $t=t+1$.

12. Generate new bat solutions $x_{i}^{t}$ by adjusting frequency as in (2), (3) and (4). 
Citation: Ali AF, Tawhid MA (2018) Solving Integer Programming Problems by Hybrid Bat Algorithm and Direct Search Method. Trends Artif Intell 2(1):46-59.

13. Update the bat velocities $v_{i}^{t}$ as in (3).

14. Calculate the new population by calculating the objective function $f\left(x_{i}^{t}\right)$ for each solution in the population.

15. Choose the best solution $x^{*}$ from the population.

16. if $\left(\right.$ rand $\left.>r_{\mathrm{i}}\right)$ then

17. Choose a solution among the best solutions.

18. Use the pattern search method in Algorithm 2 on the best solution from the best solutions list. \{exploitation process\}

\section{9. end if}

20. Generate a random new solution.

21. if $\left(\right.$ rand $\left.<A_{i}\right) \& f\left(x_{i}^{t}<f\left(x^{*}\right)\right)$ then

22. Accept the new solutions

23. Reduce the loudness $A_{0}$ and increase the rate of pulse emission $r_{i}$ and as in (5) and (6).

\section{4. end if}

25. Calculate the new population by evaluating the objective function $f\left(x_{0}^{t}\right)$ for each solution in the population.

26. Rank the population and choose the best solution $x^{*}$ from the population.

27. until $\left(t>\operatorname{Max}_{i t r}\right)$ \{Stopping criteria are satisfied.\}

28. Employ Nelder-Mead method on the best solutions, $N_{\text {elite }}$, as shown in Algorithm 3.\{Final intensification\}

Step 1: The parameters of the minimum frequency $f_{\text {min }}$, maximum frequency $f_{\text {max }}$, population size $P$, the loudness constant $\alpha$, the rate of pulse emission constant $\gamma$, the initial loudness $A_{0}$, the minimum loudness $A_{\text {min }}$, the initial rate of pulse emission $r_{0}$, the maximum num- ber of iterations $\operatorname{Max}_{i t r}$ and the initial iteration counter are set to their initial values. (Lines 1-2)

Step 2: The initial population is randomly generated by generating the initial position $x^{0}$ and the initial velocity $v^{0}$ for each bat (solution) in the population. The initial frequency $f_{i}^{0}$ is assigned to each solution in the population. The initial population is evaluated by calculating the objective function for each solution via the initial population $f\left(x_{i}^{0}\right)$ and the values of pulse rate $r_{i}$ and initial loudness $A_{i}$. (Lines 3-9)

Step 3: The new population is generated by adjusting the position $x_{i}$ and the velocity $v_{i}$ for each solution in the population as in (2), (3) and (4). (Lines 12-13)

Step 4: The new population is determined by evaluating the objective function for each solution and the best solution $x^{*}$ is selected from the population. (Lines 14-15)

Step 5: The pattern search method is considered as a local search method and used in Algorithm 2 in order to improve the best found solution at each iteration. (Lines 16-19)

Step 6: The new solution is accepted with some proximity depending on parameter $A_{i}$, increasing the rate of pulse emission and decreasing the loudness as in (5) and (6). (Lines 21-24)

Step 7: The new population is calculated, and the best solution is chosen from the population. The operations are repeated until stopping criteria are satisfied.

Step 8: The Nelder-Mead method is used on the best found solution in the previous stage as a final intensification process in order to speed up the search and avoid running the algorithm with more iterations without any enhancement. (Line 28)

\section{Numerical Experiments}

In this section, the efficiency of the HBDS algorithm

Table 2: Parameter setting.

\begin{tabular}{|l|l|l|}
\hline Parameter & Definitions & Values \\
\hline$P$ & Population size & 20 \\
\hline$f_{\min }$ & Minimum frequency & 0 \\
\hline$f_{\max }$ & Maximum frequency & 5 \\
\hline$A_{0}$ & The initial loudness & 1 \\
\hline$r_{0}$ & The initial pulse rate & 0.5 \\
\hline$\alpha$ & The loudness constant & 0.9 \\
\hline$Y$ & The rate of pulse emission constant & 0.9 \\
\hline$\varepsilon$ & Step size for checking for decent directions & $10^{-3}$ \\
\hline$m$ & Local PS repetition number & 5 \\
\hline$\Delta_{0}$ & Initial mesh size & $\left(U_{i}-L_{i}\right) / 3$ \\
\hline$\sigma$ & Reduction factor of mesh size & 0.01 \\
\hline$m$ & Local PS repetition number & 5 \\
\hline$M_{\text {ax }}$ & Maximum iterations number & $2 d$ \\
\hline$N_{\text {elite }}$ & Number of best solution for final intensification & 2 \\
\hline
\end{tabular}


Citation: Ali AF, Tawhid MA (2018) Solving Integer Programming Problems by Hybrid Bat Algorithm and Direct Search Method. Trends Artif Intell 2(1):46-59.

of iteration $\operatorname{Max}_{\text {itr }}=2 d$, where $d$ is the dimension of the problems.

Final intensification: The best obtained solutions from the BA and the pattern search method are recorded in a list in order to use the Nelder-Mead method on them, the number of the solutions in this list is called $N_{\text {elit }}$ in order to evade increasing the value of the function evaluation value, $N_{\text {elit }}=1$.

\section{Integer programming optimization test problems}

The efficiency of the HBDS algorithm has been examined on 7 benchmark integer programming problems $\left(F I_{1}-F I_{7}\right)$. The properties of the benchmark functions (the global optimal of each problem, function number, problem bound, and dimension of the problem) are outlined in Table 3 and the functions with their definitions are presented in Table 4 as follows.
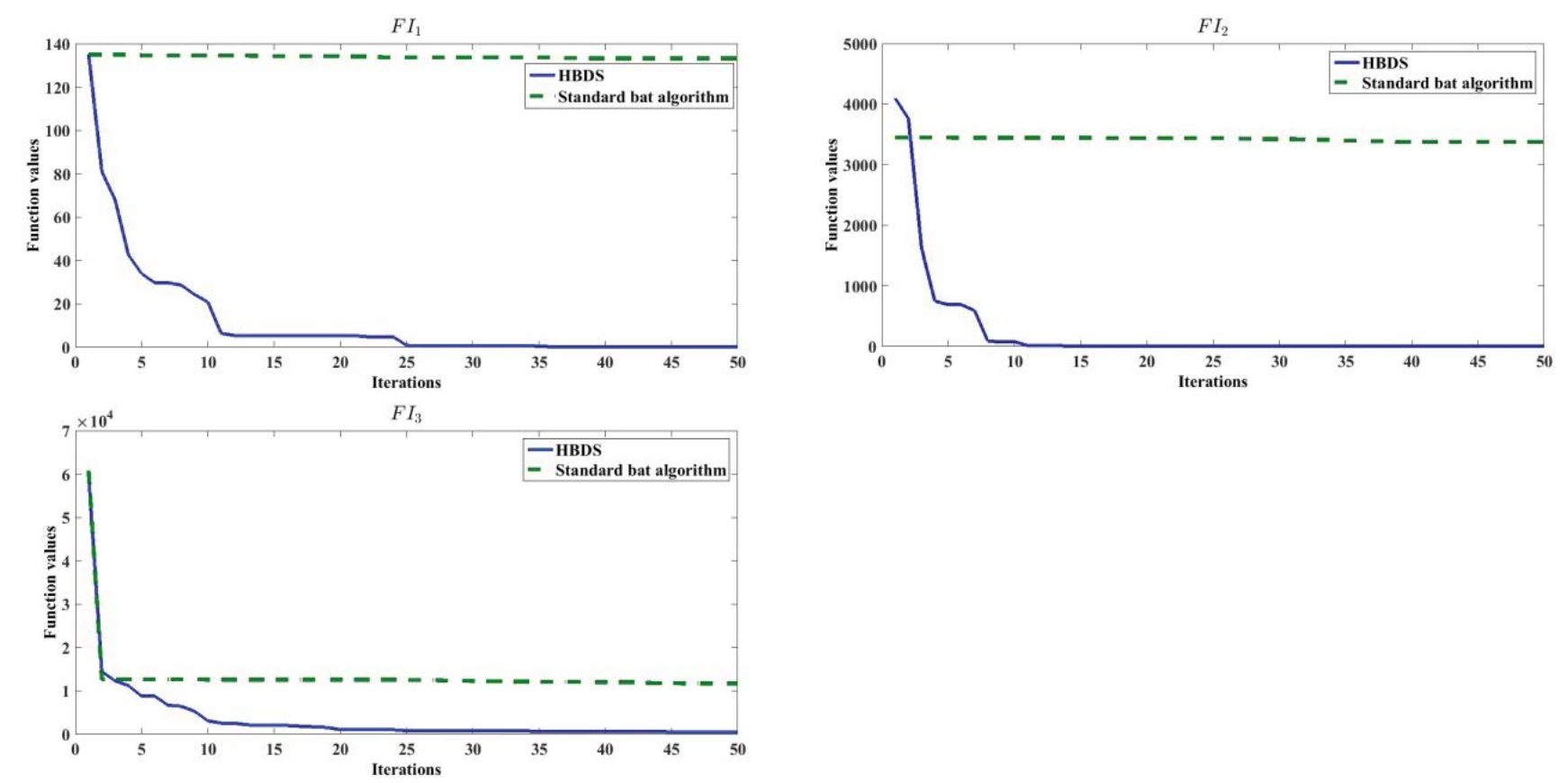

Figure 1: The efficiency of the suggested HBDS algorithm on integer programming problems.
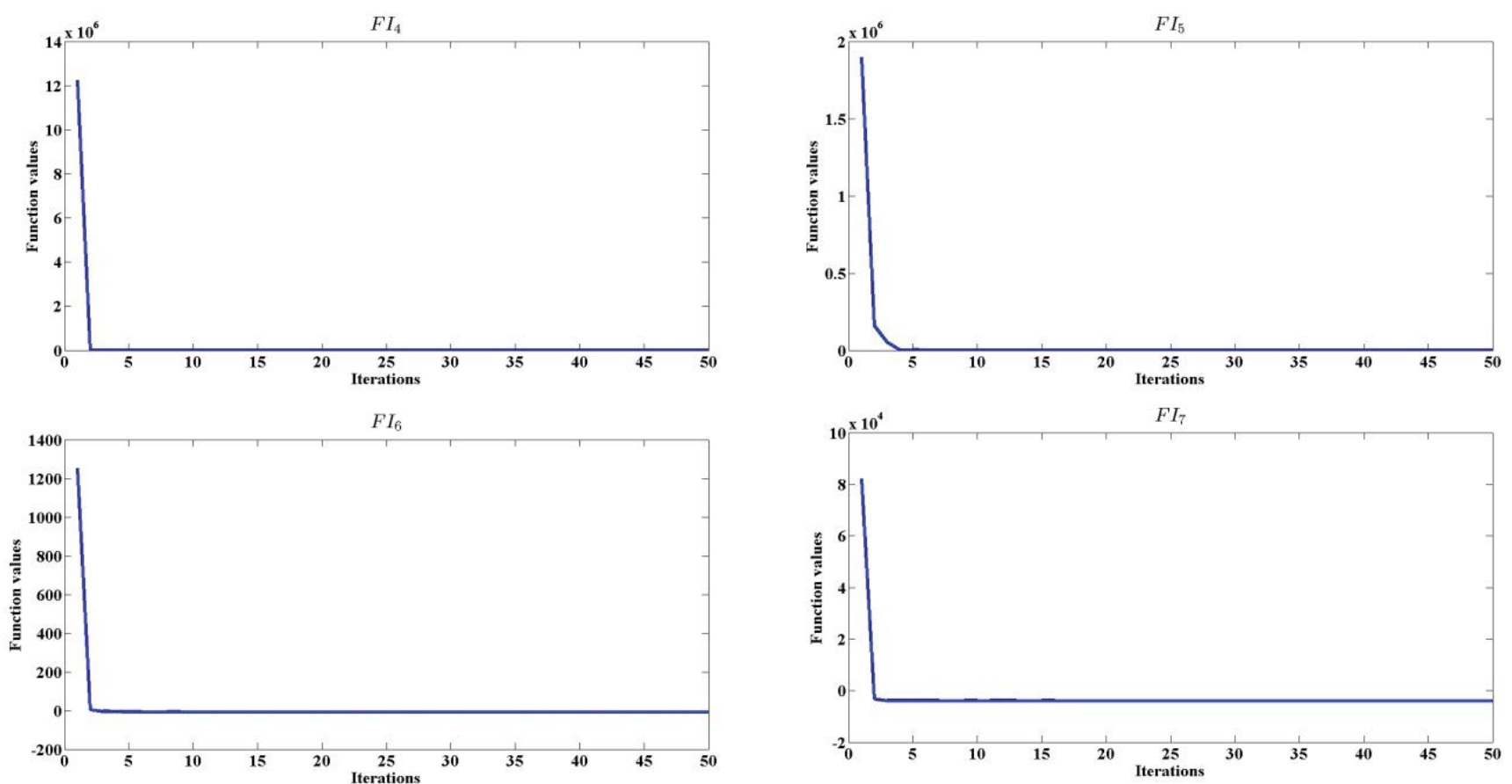

Figure 2: The performance of HBDS algorithm on integer programming problems. 
Citation: Ali AF, Tawhid MA (2018) Solving Integer Programming Problems by Hybrid Bat Algorithm and Direct Search Method. Trends Artif Intell 2(1):46-59.

\section{Comparison of HBDS without NM and HBDS with NM}

The classic BA is compared with the suggested HBDS algorithm without applying the final intensification process (Nelder-Mead method) to verify the efficiency of the suggested HBDS. The values of the parameters are designated the same for both algorithms in order to have a fair comparison. The functions $\mathrm{FI}_{1}, \mathrm{FI}_{2}$ and $\mathrm{FI}_{3}$ have been chosen to show the efficiency of the suggested algorithm by plotting the values of function values versus the number of iterations as shown in Figure 1. In Figure 1, the solid line alludes to the suggested HBDS results, while the dotted line alludes to the classic bat results after 50 iterations. Figure 1 shows that the function values rapidly reduce as the number of iterations expands for HBDS results than those of the classic BA. From Figure 1, it can be deduced that the incorporation between the classic BA with pattern search method can enhance the performance of the classic BA and speed the convergence of the suggested algorithm (Table 4).

The general performance of the suggested algorithm on the integer programming problems has been investigated by plotting the values of function values versus the number of iterations as shown in Figure 2 for four test functions $\mathrm{FI}_{4}, \mathrm{FI}_{5}, \mathrm{FI}_{6}$ and $\mathrm{FI}_{7}$. The results in Figure 2 are the results of the suggested algorithm without applying the Nelder-Mead method in the final stage of the algorithm after 50 iterations. From Figure 2, it can be deduced that the function values of the suggested HBDS rapidly reduce as the number of iterations expands and the hybridization between the BA and the pattern search method can hasten the search and help the algorithm to get the optimal or close to optimal solution in reasonable time.

The Nelder-Mead (NM) method is used in the final stage of the suggested HBDS algorithm in order to speed the convergence of the suggested algorithm and evade running the algorithm with more iterations without any enhancement in the obtained results. The results in $\mathrm{Ta}$ ble 5 show the mean evaluation function values of the suggested HBDS without and with using Nelder-Mead method, respectively. The stopping criteria mean that the

Table 5: The efficiency of calling the Nelder-Mead method in the final stage of HBDS for $\mathrm{Fl}_{1}-\mathrm{Fl}_{7}$ integer programming problems.

\begin{tabular}{|l|l|l|}
\hline Function & HBDS without NM & HBDS with NM \\
\hline $\mathrm{Fl}_{1}$ & 1800.26 & $\mathbf{6 5 6 . 5 6}$ \\
\hline $\mathrm{FI}$ & 780.13 & $\mathbf{3 4 4 . 2 2}$ \\
\hline $\mathrm{FI}_{3}$ & 20,000 & $\mathbf{1 1 3 7 . 4 8}$ \\
\hline $\mathrm{FI}_{4}$ & 410.15 & $\mathbf{2 6 0 . 8}$ \\
\hline $\mathrm{Fl}_{5}$ & 1315.45 & $\mathbf{1 1 7 7 . 1 2}$ \\
\hline $\mathrm{Fl}_{6}$ & 250.22 & 149.08 \\
\hline $\mathrm{Fl}_{7}$ & 260.15 & $\mathbf{2 2 2 . 9 1}$ \\
\hline
\end{tabular}

algorithm reaches to the global minimum of the solution within an error of $10^{-4}$ before the 20,000 function evaluation value, are designated the same for both the algorithms. The best results are outlined in boldface text. In Table 5, the results show that invoking the Nelder-Mead method in the final stage can hasten the search and help the algorithm to get the optimal or near optimal solution faster than the suggested algorithm without using the Nelder-Mead method (Table 5).

\section{HBDS and other algorithms}

The HBDS algorithm is compared with four benchmark algorithms (namely, particle swarm optimization and its variants) in order to verify of the efficiency of the suggested algorithm. Before presenting the comparison results of all algorithms, a brief description about the comparative four algorithms [24] is described.

RWMPSOg: RWMPSOg is Random Walk Memetic Particle Swarm Optimization (with global variant), which incorporates the particle swarm optimization with random walk as direction exploitation.

RWMPSOl: RWMPSOl is Random Walk Memetic Particle Swarm Optimization (with local variant), which incorporates the particle swarm optimization with random walk as direction exploitation.

PSOg: PSOg is standard particle swarm optimization with global variant without local search method.

PSOl: PSOl is standard particle swarm optimization with local variant without local search method.

Comparison between RWMPSOg, RWMPSOl, PSOg, PSOl and HBDS for integer programming problems: In this subsection, the comparison results between our HBDS algorithm and the other algorithms is given in order to validate the efficiency of our HBDS algorithm. The four comparative algorithms are examined on 7 benchmark functions. The results of the comparative algorithms are considered from their original paper [24]. The minimum ( $\min )$, maximum ( $\max$ ), average (Mean), standard deviation (St. D) and success rate (\% Suc) of the evaluation function values are outlined over 50 runs in Table 6. The run is regarded successful if the algorithm gets to the global minimum of the solution within an error of $10^{-6}$ before the 20,000 function evaluation value. The best results between the comparative algorithms are outlined in boldface text. The results in Table 6 , show that the suggested HBDS algorithm is successful in all runs and gets the objective value of each function faster than the other algorithms in 5 of 7 functions.

HBDS and other meta-heuristics and swarm intelligence algorithms for integer programming problems 
Citation: Ali AF, Tawhid MA (2018) Solving Integer Programming Problems by Hybrid Bat Algorithm and Direct Search Method. Trends Artif Intell 2(1):46-59.

Table 6: Experimental results (min, max, mean, standard deviation and rate of success) of function evaluation for $\mathrm{Fl}_{1}-\mathrm{Fl}_{7}$ test problems.

\begin{tabular}{|c|c|c|c|c|c|c|}
\hline Function & Algorithm & Min & Max & Mean & St. D & Suc \\
\hline \multirow{5}{*}{$\mathrm{Fl}_{1}$} & RWMPSOg & 17,160 & 74,699 & $27,176.3$ & 8657 & 50 \\
\hline & RWMPSOI & 24,870 & 35,265 & $30,923.9$ & 2405 & 50 \\
\hline & PSOg & 14,000 & 261,100 & $29,435.3$ & 42,039 & 34 \\
\hline & PSOI & 27,400 & 35,800 & 31,252 & 1818 & 50 \\
\hline & HBDS & 382 & 762 & 712.34 & 111.12 & 50 \\
\hline \multirow{5}{*}{$\mathrm{FI}_{2}$} & RWMPSOg & 252 & 912 & 578.5 & 136.5 & 50 \\
\hline & RWMPSOI & 369 & 1931 & 773.9 & 285.5 & 50 \\
\hline & PSOg & 400 & 1000 & 606.4 & 119 & 50 \\
\hline & PSOI & 450 & 1470 & 830.2 & 206 & 50 \\
\hline & HBDS & 295 & 412 & 375.35 & 61.45 & 50 \\
\hline \multirow[t]{5}{*}{$\mathrm{Fl}_{3}$} & RWMPSOg & 1361 & 41,593 & 6490.6 & 6913 & 50 \\
\hline & RWMPSOI & 5003 & 15,833 & 9292.6 & 2444 & 50 \\
\hline & PSOg & 2150 & 187,000 & 12,681 & 35,067 & 50 \\
\hline & PSOI & 4650 & 22,650 & 11,320 & 3803 & 50 \\
\hline & HBDS & 815 & 1315 & 1210.12 & 119.48 & 50 \\
\hline \multirow[t]{5}{*}{$\mathrm{FI}_{4}$} & RWMPSOg & 76 & 468 & 215 & 97.9 & 50 \\
\hline & RWMPSOI & 73 & 620 & 218.7 & 115.3 & 50 \\
\hline & PSOg & 100 & 620 & 369.6 & 113.2 & 50 \\
\hline & PSOI & 120 & 920 & 390 & 134.6 & 50 \\
\hline & HBDS & 258 & 311 & 275.22 & 13.22 & 50 \\
\hline \multirow[t]{5}{*}{$\mathrm{FI}_{5}$} & RWMPSOg & 687 & 2439 & 1521.8 & 360.7 & 50 \\
\hline & RWMPSOI & 675 & 3863 & 2102.9 & 689.5 & 50 \\
\hline & PSOg & 680 & 3440 & 1499 & 513.1 & 50 \\
\hline & PSOI & 800 & 3880 & 2472.4 & 637.5 & 50 \\
\hline & HBDS & 913 & 1520 & 1212.34 & 245.38 & 50 \\
\hline \multirow[t]{5}{*}{$\mathrm{FI}_{6}$} & RWMPSOg & 40 & 238 & 110.9 & 48.6 & 50 \\
\hline & RWMPSOI & 40 & 235 & 112 & 48.7 & 50 \\
\hline & PSOg & 80 & 350 & 204.8 & 62 & 50 \\
\hline & PSOI & 70 & 520 & 256 & 107.5 & 50 \\
\hline & HBDS & 140 & 175 & 152.18 & 14.25 & 50 \\
\hline \multirow[t]{5}{*}{$\mathrm{FI}_{7}$} & RWMPSOg & 72 & 620 & 242.7 & 132.2 & 50 \\
\hline & RWMPSOI & 70 & 573 & 248.9 & 134.4 & 50 \\
\hline & PSOg & 100 & 660 & 421.2 & 134.4 & 50 \\
\hline & PSOI & 100 & 820 & 466 & 165 & 50 \\
\hline & HBDS & 215 & 244.15 & 224.13 & 14.34 & 50 \\
\hline
\end{tabular}

The HBDS algorithm is investigated with various meta-heuristics and swarm intelligence algorithms (SI) such as genetic algorithm (GA) [44], standard particle swarm optimization (PSO) [8], firefly (FF) [17], cuckoo search (CS) [16] and grey wolf optimization algorithms (GWO) [45]. Having fair comparison, the population size is set 20 for all algorithms and the stopping criteria for all algorithm are the same which are the algorithm gets to the global minimum of the solution within an error of $10^{-4}$ before the 20,000 function evaluation value. For the GA the probability of crossover is set to $P C=0.8$, probability of mutation $P M=0.01$ and the roulette wheel selection is used. For the other SI algorithms, Table 6 the standard parameter setting for each algorithm is considered. The average (Avg) and standard deviation (SD) of all algo- rithms are outlined over 50 runs as shown in Table 7.

It can be concluded from Table 7 that the suggested algorithm can get the desired optimum values faster than the other SI algorithm.

\section{HBATDS and the branch and bound method}

In order to investigate the power of the HBATDS algorithm, it is compared with another famous method which is called branch and bound method (BB) [46-49]. Before discussing the comparative results between the proposed algorithm and $\mathrm{BB}$ method, the $\mathrm{BB}$ method and the main steps of its algorithm are presented.

Branch and bound method: The branch and bound method (BB) is one of the most widely used method for 
Citation: Ali AF, Tawhid MA (2018) Solving Integer Programming Problems by Hybrid Bat Algorithm and Direct Search Method. Trends Artif Intell 2(1):46-59.

Table 7: HBDS and other meta-heuristics and swarm intelligence algorithms for $\mathrm{Fl}_{1}$ - $\mathrm{Fl}_{7}$ integer programming problems.

\begin{tabular}{|l|l|l|l|l|l|l|l|}
\hline Function & & GA & PSO & FF & CS & GWO & HBDS \\
\hline$F_{1}$ & Avg SD & 1640.23 & 20,000 & 1617.13 & $11,880.15$ & 860.45 & $\mathbf{6 5 6 . 5 6}$ \\
& & 425.18 & 0.00 & 114.77 & 623.41 & 43.66 & 88.65 \\
\hline$F_{2}$ & Avg SD & 1140.15 & $17,540.17$ & 834.15 & 7176.23 & 880.25 & $\mathbf{3 4 4 . 2 2}$ \\
& & 345.25 & 1054.56 & 146.85 & 637.75 & 61.58 & 43.32 \\
\hline$F I_{3}$ & Avg SD & 4120.25 & 20,000 & 1225.17 & 6400.25 & 4940.56 & 1137.56 \\
& & 650.21 & 0.00 & 128.39 & 819.94 & 246.89 & 85.61 \\
\hline$F_{4}$ & Avg SD & 1020.35 & $16,240.36$ & 476.16 & 4920.35 & 2840.45 & $\mathbf{2 6 0 . 8}$ \\
& & 452.56 & 1484.96 & 31.29 & 247.19 & 152.35 & 10.39 \\
\hline$F I_{5}$ & Avg & 1140.75 & $13,120.45$ & 1315.53 & 7540.38 & 1620.65 & 1177.12 \\
& SD & 245.78 & 1711.83 & 113.01 & 440.82 & 111.66 & 111.6 \\
\hline$F I_{6}$ & Avg SD & 1040.45 & 1340.14 & 345.71 & 4875.35 & 3660.25 & 149.08 \\
& & 115.48 & 265.21 & 35.52 & 865.11 & 431.25 & 8.21 \\
\hline$F I_{7}$ & Avg SD & 1060.75 & 1220.46 & 675.48 & 3660.45 & 1120.15 & $\mathbf{2 2 2 . 9 1}$ \\
& & 154.89 & 177.19 & 36.36 & 383.23 & 167.54 & 11.19 \\
\hline
\end{tabular}

solving optimization problems. The main idea of $\mathrm{BB}$ method is the feasible region of the problem is subsequently partitioned into several sub regions, this operation is called branching. The lower and upper bounds value of the function can be determined over these partitions, this operation is called bounding. The main steps of $\mathrm{BB}$ method are reported in Algorithm 6, and the BB algorithm can be summarized in the following steps.

Step 1: The algorithm starts with a relaxed feasible region $M_{0} \supset S$, where $S$ is the feasible region of the problem. This feasible region $M_{0}$ is partitioned into finitely many subsets $M_{i}$.

Step 2: For each subset $M_{i}$, the lower bound $\beta$ and the upper bound $\alpha$ have been determined, where $\beta\left(M_{i}\right) \leq \inf$ $f\left(M_{i} \cap S\right) \leq \alpha\left(M_{i}\right), f$ is the objective function.

Algorithm 6: The branch and bound algorithm

1. Set the feasible region $M_{0}, M_{0} \supset S$.

2. Set $i=0$

\section{3. repeat}

4. Set $i=i+1$ $M_{i}$.

5. Partition the feasible region $M_{0}$ into many subsets

6. For each subset $M_{i}$, determine lower bound $\beta$, where $\beta=\min \beta\left(M_{i}\right)$.

7. For each subset $M_{i}$, determine upper bound $\alpha$, where $\alpha=\min \alpha\left(M_{i}\right)$.

8. if $(\alpha=\beta) \|(\alpha-\beta \leq)$ then

9. Stop

10. else

11. Select some of the subset $M_{i}$ and partition them

12. end if
13. Determine new bound on the new partition elements.

\section{4. until $(i \leq m)$}

Step 3: The algorithm is terminated, if the bounds are equal or very close, i.e. $\alpha=\beta$ (or $\alpha-\beta \leq \epsilon) \in$, is a predefined positive constant.

Step 4: Otherwise, if the bounds are not equal or very close, some of the subsets $M_{i}$ are selected and partitioned in order to obtain a more refined partition of $M_{0}$.

Step 5: The procedure is repeated until termination criteria are satisfied.

Comparison between BB method and HBATDS algorithm for integer programming problems: In this subsection, the HBATDS algorithm is tested with the $\mathrm{BB}$ method. The results of the BB method are taken from its original paper [?]. In [?], the BB algorithm transforms the initial integer problem programming problem to a continuous problem. For the bounding, the $\mathrm{BB}$ uses the sequential quadratic programming method to solve the generated sub problems, while for branching, $\mathrm{BB}$ uses depth first traversal with backtracking. In Table 8, the comparative results between the $\mathrm{BB}$ method and the proposed algorithm are reported. In Table 8, the average (Mean), standard deviation (St. D) and rate of success (Suc) are reported after 30 runs. The best mean evaluation values between the two algorithms are marked in boldface. The results in Table 8 show that the proposed algorithm results are better than the results of the $\mathrm{BB}$ method, however the BB method is better than the proposed algorithm in function $\mathrm{FI}_{2}$. The overall results in Table 8 and Figure 3 show that the proposed algorithm is faster and more efficient than the BB method.

It can be concluded from the two comparison tests between the proposed HBATDS algorithm and the 5 benchmark algorithms, that the proposed HBATDS al- 
Citation: Ali AF, Tawhid MA (2018) Solving Integer Programming Problems by Hybrid Bat Algorithm and Direct Search Method. Trends Artif Intell 2(1):46-59.
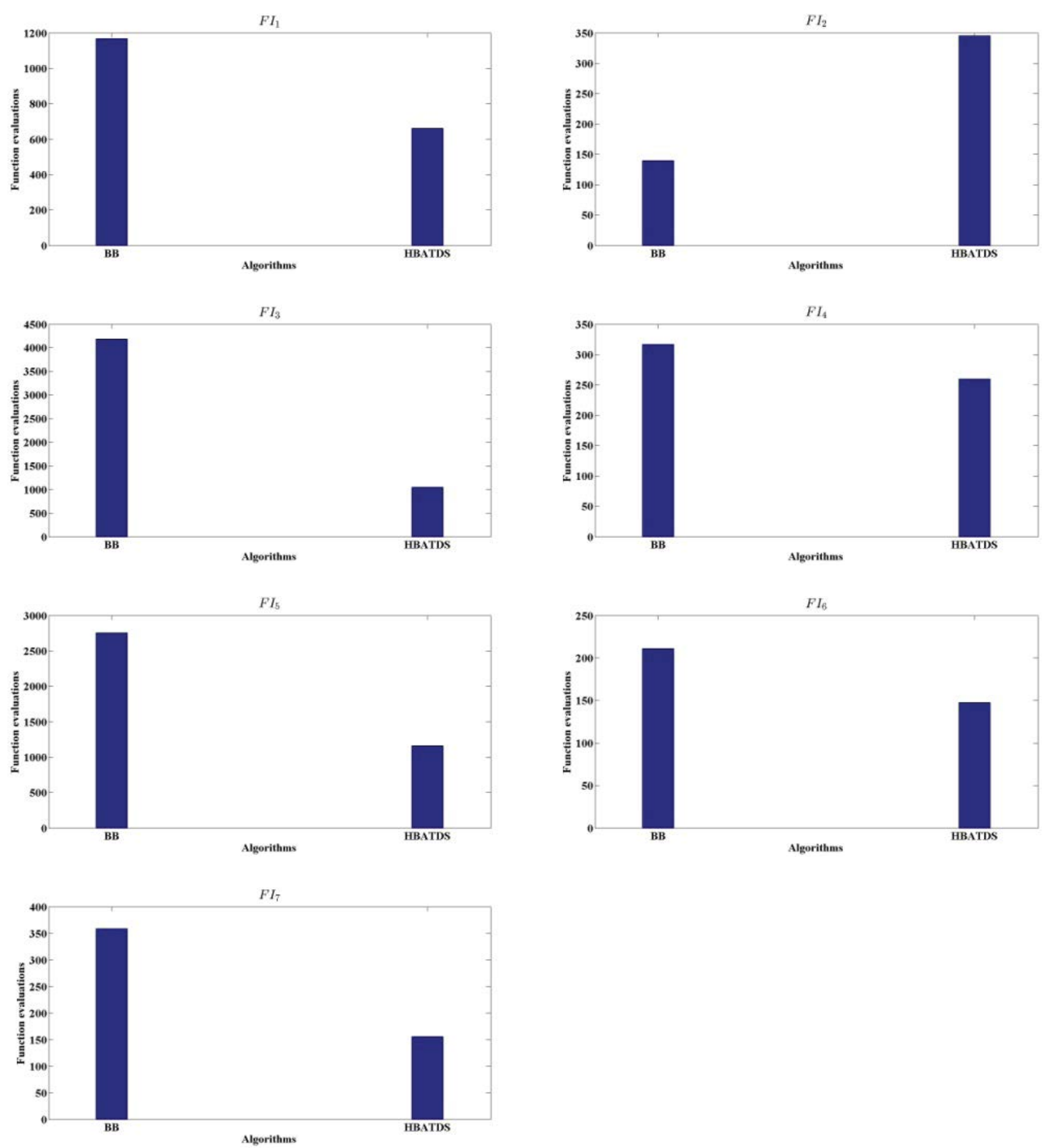

Figure 3: The comparison results (function evaluation values) between HBATDS algorithm and BB method for integer programming problems.

gorithm is a promising algorithm and can obtain the optimal or near optimal function values for most of the test functions (Table 8).

\section{Conclusion and Future Work}

In this paper, a new hybrid algorithm is suggested by incorporating the bat algorithm with direct search methods to solve integer programming problems. The suggested algorithm is named hybrid bat direct search algorithm (HBDS). In the suggested algorithm, The performance of the classic BA is accelerated by invoking the pattern search method as a local search method and the Nelder-Mead method in the final stage of the algorithm. The HBDS algorithm is intensely tested on 7 integer programming problems. The suggested algorithm is compared with other 10 algorithms to test its performance for integer programming problems. The numerical results illustrate that the suggested HBDS algorithm is a 
Citation: Ali AF, Tawhid MA (2018) Solving Integer Programming Problems by Hybrid Bat Algorithm and Direct Search Method. Trends Artif Intell 2(1):46-59.

Table 8: Experimental results (mean, standard deviation and rate of success) of function evaluation between BB method and HBATDS algorithm for $\mathrm{Fl}_{1}-\mathrm{Fl}_{7}$ test problems.

\begin{tabular}{|c|c|c|c|c|c|}
\hline Function & Algorithm & Optimal & Mean & St. D & Suc \\
\hline $\mathrm{Fl}_{1}$ & $\begin{array}{l}\text { BB } \\
\text { HBATDS }\end{array}$ & 0.00 & $\begin{array}{l}1167.83 \\
663.097\end{array}$ & $\begin{array}{l}659.8 \\
47.13\end{array}$ & $\begin{array}{l}30 \\
30\end{array}$ \\
\hline $\mathrm{Fl}_{2}$ & BB HBATDS & 0.00 & $\begin{array}{l}139.7 \\
345.64\end{array}$ & $\begin{array}{l}102.6 \\
15.75\end{array}$ & $\begin{array}{l}30 \\
30\end{array}$ \\
\hline $\mathrm{Fl}_{3}$ & BB HBATDS & -737 & $\begin{array}{l}4185.5 \\
1150.67\end{array}$ & $\begin{array}{l}32.8 \\
36.71\end{array}$ & $\begin{array}{l}30 \\
30\end{array}$ \\
\hline $\mathrm{Fl}_{4}$ & BB HBATDS & 0.00 & $\begin{array}{l}316.9 \\
265.24\end{array}$ & $\begin{array}{l}125.4 \\
4.93\end{array}$ & $\begin{array}{l}30 \\
30\end{array}$ \\
\hline $\mathrm{Fl}_{5}$ & BB HBATDS & 0.00 & $\begin{array}{l}2754 \\
1264.54\end{array}$ & $\begin{array}{l}1030.1 \\
49.14\end{array}$ & $\begin{array}{l}30 \\
30\end{array}$ \\
\hline $\mathrm{FI}_{6}$ & BB HBATDS & -6 & $\begin{array}{l}211 \\
147.77\end{array}$ & $\begin{array}{l}15 \\
4.45\end{array}$ & $\begin{array}{l}30 \\
30\end{array}$ \\
\hline $\mathrm{Fl}_{7}$ & BB HBATDS & -3833.12 & $\begin{array}{l}358.6 \\
215.48\end{array}$ & $\begin{array}{l}14.7 \\
5.14\end{array}$ & $\begin{array}{l}30 \\
30\end{array}$ \\
\hline
\end{tabular}

potential algorithm and suitable to find a global optimal solution or close optimal solution in reasonable time.

Our work in this paper motivates us to work on the proposed algorithm to solve various optimization problems such as large scale and molecular energy function [7], other combinatorial problems, MIPLIB instances, large scale integer programming and minimax problems, and constrained optimization and engineering problems [50].

\section{Acknowledgments}

We are grateful to the referees for the detailed review of our paper, insightful comments and constructive suggestions which improve the structure of the paper. The research of the $2^{\text {nd }}$ author is supported in part by the Natural Sciences and Engineering Research Council of Canada (NSERC). The postdoctoral fellowship of the $1^{\text {st }}$ author is supported by NSERC.

\section{References}

1. DZ Du, PM Pardalos (1995) Minimax and applications. Kluwer.

2. GL Nemhauser, AHG Rinnooy Kan, MJ Todd (1989) Handbooks in OR \& MS.

3. S Zuhe, A Neumaier, MC Eiermann (1990) Solving minimax problems by interval methods. BIT Numerical Mathematics 30: 742-751.

4. M Dorigo (1992) Optimization, learning and natural algorithms. Ph.D. Thesis, Politecnico di Milano, Italy.

5. X Zhang, S Wang, L Yi, et al. (2018) An integrated ant col- ony optimization algorithm to solve job allocating and tool scheduling problem. Proceedings of the Institution of Mechanical Engineers, Part B: Journal of Engineering Manufacture 232: 172-182.

6. D Karaboga, B Basturk (2007) A powerful and efficient algorithm for numerical function optimization: Artificial bee colony $(A B C)$ algorithm. Journal of Global Optimization 39: 459-471.

7. AF Ali, MA Tawhid (2017) A hybrid particle swarm optimization and genetic algorithm with population partitioning for large scale optimization problems. Ain Shams Engineering Journal 8: 191-206.

8. J Kennedy, RC Eberhart (1995) Particle swarm optimization. Proceedings of the IEEE International Conference on Neural Networks 4: 1942-1948.

9. A Sombat, T Saleewong, P Kumam (2018) Perspectives and experiments of hybrid particle swarm optimization and genetic algorithms to solve optimization problems. International Econometric Conference of Vietnam 290-297.

10. MK Passino (2002) Biomimicry of bacterial foraging for distributed optimization and control. IEEE Control Systems 22: 52-67.

11. MA Al-Betar, MA Awadallah, H Faris, et al. (2018) Bat-inspired algorithms with natural selection mechanisms for global optimization. Neurocomputing 273: 448-465.

12. XS Yang (2010) A new metaheuristic bat-inspired algorithm. Nature Inspired Cooperative Strategies for Optimization 65-74.

13. D Teodorovic, M Dell'Orco (2005) Bee colony optimization A cooperative learning approach to complex transportation problems. Advanced OR and Al Methods in Transportation 51-60. 
Citation: Ali AF, Tawhid MA (2018) Solving Integer Programming Problems by Hybrid Bat Algorithm and Direct Search Method. Trends Artif Intell 2(1):46-59.

14. R Tang, S Fong, XS Yang, et al. (2012) Wolf search algorithm with ephemeral memory. Seventh International Conference on Digital Information Management 165-172.

15. SC Chu, PW Tsai, JS Pan (2006) Cat swarm optimization. Pacific Rim International Conference on Artificial Intelligence 4099: 854-858.

16. XS Yang, S Deb (2009) Cuckoo search via Levy fights. In Proc. of World Congress on Nature \& Biologically Inspired Computing, India, IEEE Publications, USA, 210-214.

17. XS Yang (2010) Firefly algorithm, stochastic test functions and design optimization. Int J Bio-Inspired Computation 2: 78-84.

18. XL Li, ZJ Shao, JX Qian (2002) Optimizing method based on autonomous animals: Fish-swarm algorithm. Systems Engineering - Theory \& Practice 22: 32-38.

19. AF Ali, MA Tawhid (2016) Hybrid-simulated annealing and pattern search method for solving minimax and integer programming problems. Pacific Journal of Optimization 12: 51 184.

20. AF Ali, MA Tawhid (2016) Direct gravitational search algorithm for global optimization problems. East Asian Journal on Applied Mathematics 6: 290-313.

21. DS Chen, RG Batson, Y Dang (2011) Applied integer programming: Modeling and solution. John Wiley \& Sons.

22. M Conforti, G Cornujols, G Zambelli (2014) Integer programming. Graduate Texts in Mathematics.

23. MA Tawhid, AF Ali (2016) A simplex social spider algorithm for solving integer programming and minimax problems. Memetic Computing 8: 169-188.

24. YG Petalas, KE Parsopoulos, MN Vrahatis (2007) Memetic particle swarm optimization. Ann Oper Res 156: 99-127.

25. N Bacanin, I Brajevic, M Tuba (2013) Firefly algorithm applied to integer programming problems. Recent Advances in Mathematics.

26. MA Tawhid, AF Ali (2016) Direct search firefly algorithm for solving global optimization problems. App Math Inf Sci 841860.

27. AF Ali, MA Tawhid (2016) A hybrid cuckoo search algorithm with Nelder Mead method for solving global optimization problems. Springer Plus 5: 473.

28. M Tuba, M Subotic, N Stanarevic (2012) Performance of a modified cuckoo search algorithm for unconstrained optimization problems. WSEAS Transactions on Systems 11: 62-74.

29. R Jovanovic, M Tuba (2013) Ant colony optimization algorithm with pheromone correction strategy for minimum connected dominating set problem. Computer Science and Information Systems 10.

30. N Bacanin, M Tuba (2012) Artificial bee colony (ABC) algorithm for constrained optimization improved with genetic operators. Studies in Informatics and Control 21: 137-146.

31. M Tuba, N Bacanin, N Stanarevic (2012) Adjusted artificial bee colony $(A B C)$ algorithm for engineering problems. WSEAS Transaction on Computers 11: 111-120.

32. MA Tawhid, AF Ali (2016) Simplex particle swarm optimization with arithmetical crossover for solving global optimization problems. OPSEARCH 53: 705-740.

33. R Jovanovic, M Tuba (2011) An ant colony optimization algorithm with improved pheromone correction strategy for the minimum weight vertex cover problem. Applied Soft
Computing 11: 5360-5366.

34. JH Lin, CW Chou, CH Yang, et al. (2012) A chaotic levy flight bat algorithm for parameter estimation in nonlinear dynamic biological systems. Journal Computer and Information Technology 2: 56-63.

35. JW Zhang, GG Wang (2012) Image matching using a bat algorithm with mutation. Applied Mechanics and Materials 203: 88-93.

36. XS Yang (2011) Bat algorithm for multi-objective optimization. International Journal of Bio-Inspired Computation 3: 267-274.

37. G Komarasamy, A Wahi (2012) An optimized K-means clustering technique using bat algorithm. European Journal Scientific Research 84: 263-273.

38. RYM Nakamura, LAM Pereira, KA Costa, et al. (2012) BBA: A binary bat algorithm for feature selection. In: $25^{\text {th }}$ SIBGRAPI Conference on Graphics, Patterns and Images (SIBGRAPI), IEEE Publication, 291-297.

39. J Xie, Y Zhou, H Chen (2013) A novel bat algorithm based on differential operator and Levy flights trajectory. Comput Intell Neurosci 2013: 453812.

40. G Wang, L Guo (2013) A novel hybrid bat algorithm with harmony search for global numerical optimization. J Applied Mathematics 2013: 1-21.

41. FS Hillier, GJ Lieberman (1995) Introduction to operations research. MC Graw-Hill.

42. R Hooke, TA Jeeves (1961) Direct search, solution of numerical and statistical problems. J Ass Comput 8: 212-229.

43. JA Nelder, R Mead (1965) A simplex method for function minimization. Computer Journal 7: 308-313.

44. JH Holland (1975) Adaptation in natural and artificial systems. University of Michigan Press, Ann Arbor, Michigan.

45. S Mirjalili, SM Mirjalili, A Lewis (2014) Grey wolf optimizer. Advances in Engineering Software 69: 46-61.

46. B Borchers, JE Mitchell (1992) Using an interior point method in a branch and bound algorithm for integer programming. Technical Report, Rensselaer Polytechnic Institute.

47. B Borchers, JE Mitchell (1994) An improved branch and bound algorithm for mixed integer nonlinear programs. Computers \& Operations Research 21: 359-367.

48. EL Lawler, DW Wood (1966) Branch and bound methods: A Survey. Operations Research 14: 699-719.

49. VM Manquinho, JP Marques Silva, AL Oliveira, et al. (1997) Branch and bound algorithms for highly constrained integer programs. Technical Report, Cadence European Laboratories, Portugal.

50. AF Ali, MA Tawhid (2016) Hybrid PSO and DE algorithm for solving engineering optimization problems. Applied Mathematics and Information Sciences 10: 431-449.

51. G Rudolph (1994) An evolutionary algorithm for integer programming. In: Davidor Y, Schwefel HP, Mnner R, Parallel Problem Solving from Nature. 3: 139-148.

52. A Glankwahmdee, JS Liebman, GL Hogg (1979) Unconstrained discrete nonlinear programming. Engineering Optimization 4: 95-107.

53. SS Rao (1994) Engineering optimization-theory and practice. $\left(4^{\text {th }}\right.$ edn), Wiley, New Delhi, India. 